\title{
Pré e Pós operatório nas DII \\ A Visão do Cirurgião
}

\section{André da Luz Moreira}

\section{Introdução}

A maioria dos pacientes com doença de Crohn (DC) necessitam de pelo menos um procedimento cirúrgico durante o curso natural da doença, frequentemente por estenoses ou fistulas. 0 risco cumulativo de ressecção intestinal na DC pode chegar a 70-80\% em 10 anos após o diagnostico. Na retocolite ulcerativa (RCUI), aproximadamente $30-40 \%$ dos pacientes são submetidos a cirurgia por doença refratária ao tratamento clínico, e em menor escala por displasia, neoplasia ou colite aguda grave.

Em grande parte das vezes, o tratamento clinico é levado ao extremo e a cirurgia somente é recomendada em ultimo caso. Consequentemente, no pré-operatório, os pacientes podem se apresentar desnutridos, imunossuprimidos e alguns com sinais de sepse intra-abdominal. Esses fatores estão associados a um risco aumentado de inúmeras complicações pós-operatórias e apresentam um grande desafio para o cirurgião. Portanto, a otimização pré-operatória na tentativa de diminuir esses riscos e trazer bons resultados cirúrgicos é imperativa na avaliação desses pacientes.

\section{Avaliação Nutricional}

A desnutrição pré-operatória aumenta as taxas de morbidade pós-operatória e o risco de sepse abdominal. Muitos pacientes são enviados ao cirurgião como último recurso e portanto chegam desnutridos por debilidade crônica. Entretanto, não existe um teste padrão para diagnosticar a desnutrição, uma vez que ela envolve uma avaliação com- 
plexa incluindo vários fatores. As deficiências nutricionais encontradas são resultado de uma ingesta inadequada de nutrientes devido a dor abdominal pós-prandial ou diarreia, má absorção devido as alterações da mucosa, a inflamação sistêmica que altera a síntese de proteínas e aumenta o catabolismo proteico e a efeitos colaterais de algumas medicações utilizadas no tratamento da doença inflamatória intestinal (DII). A complicação mais temida no pós-operatória é a deiscência de anastomose. Existem inúmeros estudos relacionando essa complicação com a hipoalbuminemia. Portanto, a otimização nutricional pré-operatória pode corrigir essa deficiência utilizando nutrição parenteral total (NPT) ou nutrição enteral (NE). A NPT administrada por pelo menos 20 a 30 dias antes da cirurgia pode restabelecer alguns parâmetros nutricionais e preparar o paciente metabolicamente para o trauma cirúrgico, reduzindo assim as complicações pós-operatórias. O suporte enteral (ex. dietas elementares ou oligomonoméricas) deve ser a via de preferência sempre que possível, por ser a via mais fisiológica, de menor custo, e com menos risco de sepse quando comparada a NPT.

\section{Manejo Farmacológico}

O principal tratamento clínico na DIl envolve a supressão da resposta imunológica utilizando corticoides, imunomoduladores e agentes biológicos. Entretanto, esses medicamentos podem estar associados a complicações pós-operatórias apesar de muitas evidências na literatura serem conflitantes.

Muitos pacientes com DII precisam de corticoides durante o curso da doença, 30-40\% desses pacientes com doença moderada ou grave ficam cortico-dependentes. Embora o cenário ideal fosse realizar uma cirurgia eletiva em pacientes que conseguiram descontinuar a corticoterapia por períodos prolongados, a realidade é que uma parcela significativa de pacientes chegam a cirurgia com doses variadas de corticoide devido a impossibilidade de suspender essa medicação a tempo. Durante o trauma cirúrgico, o organismo necessita de corticóides endógenos (resposta metabólica ao trauma) e essa resposta pode estar bloqueada pelo uso crônico de corticoides exógenos. Pacientes com insuficiência adrenal secundária à esse uso podem desenvolver hipotensão, taquicardia ou hipoglicemia no período pós-operatório uma vez que seu corpo não consegue compensar o estresse associado a 
cirurgia. Portanto, o uso de corticoides no perioperatório se justifica como uma medida preemptiva ou preventiva nos pacientes com risco de supressão do eixo Hipotalâmico-Hipofisário-Adrenal (HHA). Muito embora o grau de supressão possa estar associado tradicionalmente a dose e a duração da terapia, há poucos dados na literatura para apoiar essa teoria. Glicocorticóides equivalentes a $5 \mathrm{mg}$ de prednisona diários ou menos e administrados por menos de 3 semanas não parecem causar supressão clinica significativa do eixo HHA. Entretanto, os pacientes que receberam o equivalente a $15 \mathrm{mg}$ de prednisolona diário por mais de 3 semanas são suspeitos de ter essa supressão. Apesar de ser possível avaliar a supressão do eixo HHA através do teste da corticotropina, uma cobertura empírica para os pacientes de risco é uma abordagem mais prática na rotina dos cuidados peri-operatórios.

Os imunomoduladores também são frequentemente utilizados para manter remissão e poupar corticoide no tratamento clínico da DII. Assim como os corticoides, também há opiniões conflitantes em relação ao risco de complicações cirúrgicas em pacientes utilizando imunomoduladores, principalmente em relação a droga mais utilizada, a azatioprina. A recomendação atual sugere manter a azatioprina no período perioperatório, suspendendo no dia da cirurgia e reiniciando assim que o paciente esteja tolerando dieta oral.

Os agentes biológicos tem sido cada vez mais utilizados na DII e hoje em dia é comum a realização de cirurgias em pacientes recebendo anticorpos anti-TNF alfa. 0 uso desses agentes tem preocupado os cirurgiões em relação ao risco de complicações sépticas no pós-operatório. O risco de complicações em pacientes com DC ainda é um assunto controverso e o intervalo seguro ainda não foi determinado. Alguns autores recomendam a realização da cirurgia após 2 meses da ultima dose e o reinicio da terapia para profilaxia pós-operatória após 2 a 4 semanas da data da cirurgia. Na cirurgia para RCUI o uso de biológicos tem sido associado a um maior risco de complicações pós-operatórias, sugerindo uma maior utilização de três estágios na confecção de reservatórios íleo-anais.

\section{Manejo da Sepse}

A sepse abdominal na forma de fístulas e abscessos é relativamente comum na DC, ocorrendo em 10 a 30\% dos casos e represen- 
ta um fator de risco independente para complicações pós-operatórias. Tradicionalmente, os abscessos eram drenados cirurgicamente e a formação de estomas era frequente. Entretanto, atualmente, recomenda-se, sempre que possível, o uso de antibióticos parenterais associados ou não a drenagem percutânea guiada por imagem radiológica na tentativa de "esfriar" o processo infeccioso e evitar uma cirurgia na fase aguda, permitindo assim, uma intervenção cirúrgica eletiva com menos chance de realização de estomas.

\section{Profilaxia de Fenômenos Tromboembólicos}

Como em todas as cirurgias abdominais e pélvicas de grande porte, a profilaxia de complicações tromboembólicas deve ser realizada segundo os diretrizes sugeridas pelo the American College of Chest Physicians Evidence- Based Clinical Practice Guidelines (9th edition). 0 risco de trombose venosa profunda e embolia pulmonar está aumentado nos pacientes com DIl em até 3 vezes quando comparados com a população geral. Portanto, a profilaxia farmacológica pode não ser suficiente. Consequentemente, pacientes com DIl necessitando de cirurgia estão em alto risco para complicações tromboembólicas pós-operatórias e a profilaxia combinada, farmacológica e mecânica, deve ser considerada.

\section{Cuidados Pós-operatórios}

A profilaxia antibiótica deve ser suspensa nas primeiras 24 horas depois da cirurgia, ao menos que o paciente já tenha complicações sépticas abdominais prévias. Não há obrigatoriedade do uso de sonda nasogástrica após as cirurgias eletivas e a utilização de drenos abdominais deve ser restrito dependendo dos critérios de cada cirurgião. A dieta oral pode ser reiniciada precocemente principalmente nos casos em que a via laparoscópica foi utilizada associado a deambulação precoce e fisioterapia respiratória. A presença de enfermeiros especializados (estomaterapeutas) é importante nos pacientes submetidos a ostomias, auxiliando nos cuidados e na educação do paciente. Lembrando sempre, que o local do estoma deve sempre ser marcado no pré-operatório com o paciente acordado para melhor resultados funcionais e de qualidade de vida. 


\section{Referências bibliográficas}

1. Loftus EV Jr, Schoenfeld P, Sandborn WJ. The epidemiology and natural history of Crohn's disease in population-based patient cohorts from North America: a systematic review. Aliment Pharmacol Ther 2002;16(1):51-60

2. Yamamoto T, Allan RN, Keighley MRB. Risk factors for intraabdominal sepsis after surgery in Crohn's disease. Dis Colon Rectum 2000;43(8):1141-1145

3. White JV, Guenter P, Jensen G, Malone A, Schofield M; Academy MalnutritionWork Group A.S.P.E.N. Malnutrition Task Force A.S.P. E.N. Board of Directors. Consensus statement: Academy of Nutrition and Dietetics and American Society for Parenteral and Enteral Nutrition: characteristics recommended for the identification and documentation of adult malnutrition (undernutrition). JPEN J Parenter Enteral Nutr 2012;36(3):275-283

4. Schlaghecke R, Kornely E, Santen RT, Ridderskamp P. The effect of long-term glucocorticoid therapy on pituitary-adrenal responses to exogenous corticotropin-releasing hormone. N Engl J Med 1992;326(4):226-230

5. Bernstein CN, Blanchard JF, Houston DS, Wajda A. The incidence of deep venous thrombosis and pulmonary embolism among patients with inflammatory bowel disease: a population-based cohort study. Thromb Haemost 2001;85(3):430-434.

6. Zerbib P, Koriche D, Truant S, et al. Pre-operative management is associated with low rate of post-operative morbidity in penetrating Crohn's disease. Aliment Pharmacol Ther 2010;32(3):459465.

7. Colombel JF, Loftus EV Jr, Tremaine WJ, et al. Early postoperative complications are not increased in patients with Crohn's disease treated perioperatively with infliximab or immunosuppressive therapy. Am J Gastroenterol 2004;99(5):878-883 